\title{
NP-1250, an ABCG2 inhibitor, induces apoptotic cell death in mitoxantrone-resistant breast carcinoma MCF7 cells via a caspase-independent pathway
}

\author{
MASUMI ITO ${ }^{1,2}$, KAZUNORI KAJINO ${ }^{1}$, MASAAKI ABE ${ }^{1}$, TSUTOMU FUJIMURA ${ }^{3}$, REIKO MINEKI ${ }^{3}$, \\ TAKAKO IKEGAMI $^{4}$, TOSHIHISA ISHIKAWA ${ }^{5}$ and OKIO HINO ${ }^{1}$ \\ ${ }^{1}$ Department of Pathology and Oncology, Juntendo University School of Medicine, Bunkyo, Tokyo 113-8421; \\ ${ }^{2}$ Nippon Chemiphar Co., Ltd., Saitama 341-0005; ${ }^{3}$ Laboratory of Proteomics and Biomolecular Science, \\ Biomedical Research Center, Juntendo University Graduate School of Medicine, Bunkyo, Tokyo 113-8421; \\ ${ }^{4}$ Laboratory of Molecular and Biochemical Research, Research Support Center, Juntendo University \\ Graduate School of Medicine, Bunkyo, Tokyo 113-8421; ${ }^{5}$ Omics Science Center, \\ RIKEN Yokohama Institute, Yokohama 230-0045, Japan
}

Received September 10, 2012; Accepted November 9, 2012

DOI: $10.3892 / o r .2013 .2249$

\begin{abstract}
Chemoresistance is one of the main obstacles to successful cancer therapy and is frequently associated with multidrug resistance (MDR). One of the most studied mechanisms of MDR is the high expression of ATP-binding cassette (ABC) transporters. Here, we demonstrated that NP-1250, an ABCG2 inhibitor, induced apoptotic cell death in ABCG2-overexpressing multidrug-resistant MCF7/ mitoxantrone-resistant (MX) human breast carcinoma cells via a caspase-independent pathway. Incubation of MCF7/MX cells with NP-1250 significantly reduced cell viability, while NP-1250 had little effect on the viability of drug-sensitive MCF7/wild-type cells. Although the target molecules of NP-1250 in cell death remain unknown, investigation of NP-1250 will aid in the elucidation of the molecular mechanism of drug resistance and NP-1250 may become a new therapy for MDR cancers.
\end{abstract}

Correspondence to: Dr Okio Hino, Department of Pathology and Oncology, Juntendo University School of Medicine, 2-1-1 Hongo, Bunkyo, Tokyo 113-8421, Japan

E-mail: ohino@juntendo.ac.jp

Abbreviations: ABC, ATP-binding cassette; Flp-In-293/ABCG2 cells, ABCG2-overexpressing Flp-In-293 cells; Flp-In-293/Mock cells, mock-transfected Flp-In-293 cells; MCF7/MX cells, mitoxantroneresistant MCF7 cells; MCF7/WT cells, wild-type MCF7 cells; MDR, multidrug resistance; MTX, methotrexate; PARP, poly(ADPribose) polymerase; PDIA4, protein disulfide-isomerase A4; PHB2, prohibitin-2; PRDX2, peroxiredoxin-2

Key words: breast cancer, mitoxantrone resistance, caspaseindependent cell death, ABCG2, apoptosis

\section{Introduction}

A frequent problem in cancer chemotherapy is the development of multidrug resistance (MDR) which renders tumors unresponsive to a diverse array of compounds. There are a number of mechanisms by which a cell can acquire MDR, one of the most common being overexpression of members of the ATP-binding cassette (ABC) transporter family. This is a group of plasma membrane proteins that actively extrude a broad range of substrates from cells. They are predominantly found in areas such as the epithelial cells of the intestine, hepatocytes, capillary epithelial cells of the blood-brain barrier, and in various stem cells where the physiological role of these ATPase efflux 'pumps' is to protect cells from damage by rapidly extruding xenobiotics (1). Unfortunately, their unwanted expression in tumor cells can lead to chemotherapy resistance in these cells (2).

Most research concerning ABC transporter-overexpressing MDR cancer cells have focused on molecules or signaling pathways that regulate expression of these transporters $(3,4)$, and a few potential therapeutic target molecules have been identified. In recent years, it has been reported that suppression of ABC transporters inhibits cancer cell proliferation, particularly in transporter-overexpressing MDR cancer cells $(5,6)$. However, there are no reports concerning the relationship between inhibition of ABC transporters and cell death.

In the present study, we demonstrated that treatment with NP-1250, an ABCG2 inhibitor, alone induced apoptotic cell death via a caspase-independent pathway in MDR MCF7 breast cancer cells (MCF7/MX). MCF7/MX cells (1) have the MDR phenotype characterized by high levels of the ABCG2 transporter. We speculated that NP-1250 would have an effect on molecules involved in growth and survival, whose expression levels have been elevated in the process of acquiring resistance to anticancer drugs. Using a proteomics approach, we identified three candidate molecules: peroxiredoxin-2, 
protein disulfide-isomerase A4 and prohibitin-2. These molecules may be potential candidates for the treatment of MDR cancers.

\section{Materials and methods}

Materials. Pan-caspase inhibitor (Z-VAD-FMK) and caspase-3/7-specific inhibitor (Ac-DEVD-CHO) were purchased from Promega (Madison, WI, USA). Doxorubicin was obtained from Kyowa Hakko Kirin (Tokyo, Japan) and mitoxantrone hydrochloride was obtained from Wyeth Lederle Japan, Ltd. (Tokyo, Japan). NP-1250 was synthesized at Nippon Chemiphar Co., Ltd. (Saitama, Japan). It is still under development, therefore, disclosure of the structure of this compound is prohibited.

Cell cultures and treatment. The MCF7 human breast cancer cell line, the HL-60 human promyelocytic leukemia cell line, the multidrug-resistant HL-60/MX1 cell line, the MES-SA human uterus sarcoma cell line, and the multidrug-resistant MES-SA/Mx 2 and MES-SA/Dx 5 cancer cell lines were obtained from American Type Culture Collection (ATCC, Rockville, MD, USA) and cultured in the recommended medium containing $10 \%$ heat-inactivated fetal bovine serum (FBS) at $37^{\circ} \mathrm{C}$ in a $95 \%$ air $/ 5 \% \mathrm{CO}_{2}$ atmosphere. Mitoxantroneresistant MCF7 (MCF7/MX) cells (7) were a gift from Dr Masayuki Nakagawa (Department of Urology, Kagoshima University, Kagoshima, Japan) and doxorubicin-resistant MCF7 (MCF7/DOX) cells were a gift from Dr Takao Yamori (Division of Molecular Pharmacology, Cancer Chemotherapy Center, Japanese Foundation for Cancer Research, Tokyo, Japan). Prior to any experiments, MCF7/MX cells and MCF7/ DOX cells were maintained in drug-free medium for one passage. Other resistant cells were cultured in the absence of anticancer drugs. ABCG2-overexpressing Flp-In-293 (Flp-In-293/ABCG2) cells and mock-transfected Flp-In-293 (Flp-In-293/Mock) cells were established and cultured as previously described (8).

Evaluation of the inhibitory effect on the ABCG2 transporter. To evaluate ABCG2-NP-1250 interaction, plasma membrane vesicles prepared from ABCG2-expressing Sf9 cells were incubated with $\left[{ }^{3} \mathrm{H}\right]$ methotrexate (MTX). Transport into the vesicles was measured by counting the radioactivity remaining on the filter of MultiScreen plates, as previously described (9).

Cell viability assay. To evaluate the effectiveness of NP-1250 in overcoming ABCG2-mediated drug resistance, Flp-In293/ABCG2 and MCF7/MX cells were incubated with anticancer drug SN-38 and NP-1250, and the cell viabilities were measured.

Tumor cells were seeded in 96-well plates. After $24 \mathrm{~h}$, the medium was replaced with medium containing different concentrations of NP-1250 in triplicate. After a 72-h incubation at $37^{\circ} \mathrm{C}$, cell viability was determined at the indicated times, as previously described (10).

TUNEL staining. MCF7/WT and MCF7/MX cells were seeded in 35-mm glass-bottom dishes. After $24 \mathrm{~h}$, the medium was replaced with medium containing 10,20 or $40 \mu \mathrm{M}$ of NP-1250. After a 48 -h incubation at $37^{\circ} \mathrm{C}$, TUNEL staining was performed as previously described (10). The frequency (\%) of TUNEL-positive cells per DAPI-positive cells was determined in five different fields in duplicate. Two replicate experiments were performed.

Annexin V/PI staining. The Annexin V-FITC fluorescence microscopy kit (Pharmingen, San Diego, CA, USA) was used to detect apoptosis. MCF7/MX cells were seeded in 35-mm glass-bottom dishes. After $6,12,24$ or $48 \mathrm{~h}$ of incubation with $20 \mu \mathrm{M}$ of $\mathrm{NP}-1250$ at $37^{\circ} \mathrm{C}$, Annexin V/propidium iodide (PI) staining was performed according to the manufacturer's protocol. Stained cells were visualized using a confocal microscope (Leica).

RNA isolation and cDNA synthesis. Total RNA was prepared using the FastPure ${ }^{\circledR}$ RNA kit (9190; Takara, Shiga, Japan) according to the manufacturer's protocol, and RNA concentrations were measured at an absorbance $\mathrm{A}_{260}$. Reverse transcription (RT) was carried out with $1 \mu \mathrm{g}$ RNA using a ReverTraAce ${ }^{\circledR}$ qPCR RT kit (FSQ-101; Toyobo, Osaka, Japan).

Real-time PCR. Real-time PCR was carried out using Fast SYBR-Green Master Mix (4385612, Applied Biosystems, Life Technologies Corp., Carlsbad, CA, USA) using a 7500 Fast Real-Time PCR system (Applied Biosystems). All primers used for real-time PCR are described in Table I. PCR was carried out with an initial 20 -sec denaturation at $95^{\circ} \mathrm{C}$ followed by 40 cycles of PCR ( $3 \mathrm{sec}$ at $95^{\circ} \mathrm{C}$ and $30 \mathrm{sec}$ at $60^{\circ} \mathrm{C}$ ). Reactions were performed in triplicate for three biological replicates, and the amount of each cDNA was normalized to GAPDH gene expression. Melt-curve analysis was performed to ensure that the mRNA-specific fragments were amplified and data were analyzed using the standard curve method.

Immunofluorescence staining of $A B C G 2$. Cells were fixed in $4 \%$ paraformaldehyde, permeabilized with $0.2 \%$ Triton $\mathrm{X}-100$, and blocked with $1 \%$ bovine serum albumin and $10 \%$ normal goat serum in phosphate-buffered saline (PBS). Incubation with primary monoclonal antibody for ABCG2 (ALX-801-029; Wako, Osaka, Japan) at a dilution of 1:200 was carried out overnight at $4^{\circ} \mathrm{C}$, followed by incubation with the Alexa Fluor-conjugated secondary antibody (A11001; Molecular Probes, Inc., Carlsbad, CA, USA) for $1 \mathrm{~h}$ at room temperature. Immunofluorescence was detected and analyzed using a Leica TCS SP5 Laser Scanning Confocal Microscope system.

Western blot analysis. Cells were washed two times with PBS, harvested by scraping from the culture dishes in lysis buffer and collected. Protein concentrations were determined using the DC protein assay kit according to the manufacturer's instructions (500-0116; Bio-Rad, Hercules, CA, USA). Equal amounts of proteins were denatured by boiling and subjected to SDS-polyacrylamide gel electrophoresis. The separated proteins were transferred to polyvinylidene difluoride membranes. The membranes were incubated overnight at $4^{\circ} \mathrm{C}$ with primary polyclonal antibody at a dilution of 1:1000 for caspase-3, cleaved-PARP, cleaved-caspase-7 or cleavedcaspase-9 (9665, 9541, 9491, 9501; Cell Signaling Technology, 
Table I. Sequence of primers for real-time PCR.

\begin{tabular}{|c|c|c|c|}
\hline Gene & Direction & Primer sequence (5'-3') & Product size (bp) \\
\hline \multirow[t]{2}{*}{ GAPDH } & Forward & GCCATCAATGACCCCTTC & 114 \\
\hline & Reverse & GATGACAAGCTTCCCGTTC & \\
\hline \multirow[t]{2}{*}{ ABCB1 } & Forward & AACTTCCGAACCGTTGTTTC & 110 \\
\hline & Reverse & CCAAAGATGTGTGCTTTCCTC & \\
\hline \multirow[t]{2}{*}{$\mathrm{ABCC} 1$} & Forward & CTACCTCCTGTGGCTGAATC & 150 \\
\hline & Reverse & ATCAGCTTGATCCGATTGTC & \\
\hline \multirow[t]{2}{*}{ ABCG2 } & Forward & ACAGGTGGAGGCAAATCTTC & 94 \\
\hline & Reverse & GCGGTGCTCCATTTATCAG & \\
\hline \multirow[t]{2}{*}{ PRDX1 } & Forward & CTGTCATCTAGCATGGGTCAAT & 106 \\
\hline & Reverse & CCCATAATCCTGAGCAATG & \\
\hline \multirow[t]{2}{*}{ PRDX2 } & Forward & GTCCGTGCGTCTAGCCTTTG & 128 \\
\hline & Reverse & CAGCTTCACCTCTTTGAAGG & \\
\hline \multirow[t]{2}{*}{ LGUL } & Forward & CCAAGGATTTTCTATTGCAG & 97 \\
\hline & Reverse & TGGATTAGCGTCATTCCAAGA & \\
\hline \multirow[t]{2}{*}{ GLTP } & Forward & CCCTTCTTCGATTGCCTTG & 107 \\
\hline & Reverse & AACTTGGCTGGGTTGGTGTC & \\
\hline \multirow[t]{2}{*}{ TAGL2 } & Forward & CCAAACTGTGGACCTCTGG & 143 \\
\hline & Reverse & GATTCTCCTTGGATTTCTTAGGG & \\
\hline \multirow[t]{2}{*}{ PSB5 } & Forward & CCACCCTGGCCTTCAAGTTC & 132 \\
\hline & Reverse & CCATGGTGCCTAGCAGGTATG & \\
\hline \multirow[t]{2}{*}{ PEBP1 } & Forward & GCCCACCCAGGTTAAGAATAG & 145 \\
\hline & Reverse & GACCACCAGGAAATGATGC & \\
\hline \multirow[t]{2}{*}{ GNA1 } & Forward & CCTGTGCTAAGAGAGGAAGAGT & 143 \\
\hline & Reverse & TGGTAGACATTCAAGGGTAATC & \\
\hline \multirow[t]{2}{*}{ DCTP1 } & Forward & CTTTCAGTGGAAAACCGATG & 144 \\
\hline & Reverse & ACTGCTAGCGGCAGATCCAC & \\
\hline \multirow[t]{2}{*}{ VDAC2 } & Forward & GATTTGGTTTTGGGTTGGTG & 115 \\
\hline & Reverse & TCCAAGGTCCCAGTAACTTT & \\
\hline \multirow[t]{2}{*}{ P5CR1 } & Forward & TGGACCTGGCCACAGTTT & 103 \\
\hline & Reverse & TTCACAGCCAGGAAGAGCACAT & \\
\hline \multirow[t]{2}{*}{ SFXN1 } & Forward & TTGGCTTCTGTTTGGTGTTT & 113 \\
\hline & Reverse & GCTCTCTTGGATCTTAGCTTGC & \\
\hline \multirow[t]{2}{*}{ PDIA4 } & Forward & CCGCTAACAACCTGAGAGAAG & 105 \\
\hline & Reverse & CAGGCTGCATTACAACCAAC & \\
\hline \multirow[t]{2}{*}{ HNRPM } & Forward & AAGGGTGAAGGAGAACGACCTG & 111 \\
\hline & Reverse & GGCTCTGTATCTTTTAGTTGGA & \\
\hline \multirow[t]{2}{*}{ ECHA } & Forward & AACTCTCCCAATTCAAAGGT & 117 \\
\hline & Reverse & TGATGAGATAAGGACGGCACT & \\
\hline \multirow[t]{2}{*}{ GPDM } & Forward & TCACCAGAGGACTAAAAACAGC & 91 \\
\hline & Reverse & ACACCACCATGGATCAATTT & \\
\hline \multirow[t]{2}{*}{ IPYR } & Forward & AATGGAGATTGCTACAAAGGAC & 142 \\
\hline & Reverse & TGGGTCTTCCCAAGTCTG & \\
\hline \multirow[t]{2}{*}{ OTUB1 } & Forward & CAGCGGTTCAAGGCTGTGT & 133 \\
\hline & Reverse & CGACAGAGGTCTGCTTCTCC & \\
\hline PHB2 & Forward & CTCCAAAGACCTACAGATGGTG & 130 \\
\hline & Reverse & GTTGACAATGGACGGCAACA & \\
\hline GGCT & Forward & CCGCCTGCAGGATTTTAAG & 98 \\
\hline & Reverse & CGCCAGGACTCTGAAAAATG & \\
\hline LDHA & Forward & CTGTCATGGGTGGGTCCTT & 106 \\
\hline & Reverse & CCCTAAATCTGGGTGCAGAGTC & \\
\hline
\end{tabular}



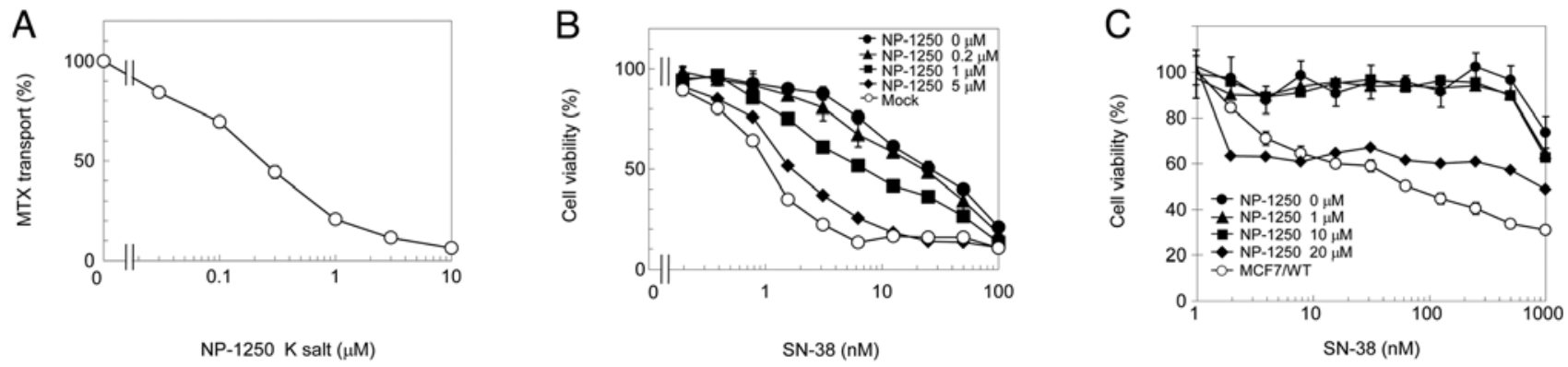

Figure 1. Inhibitory effect of NP-1250 on the ABCG2 transporter. (A) Inhibition of ABCG2-mediated [ $\left.{ }^{3} \mathrm{H}\right] \mathrm{MTX}$ transport by NP-1250. MTX transport is expressed as relative values compared with the transport activity measured without NP-1250 (100\% MTX transport). Data points, means; bars, SD ( $\mathrm{n}=4$ ). (B and C) Reversal of ABCG2-mediated SN-38 resistance by NP-1250. NP-1250 dose-dependently reversed SN-38 resistance in Flp-In-293/ABCG2 cells (B) but had no similar effect in MCF7/MX cells (C). However, in MCF7MX cells (C), $20 \mu \mathrm{M}$ of NP-1250 inhibited cell viability with a very low dose of SN-38. Data points, means; bars, $\mathrm{SD}(\mathrm{n}=4)$.

Beverly, MA, USA). Membranes were then incubated with goat anti-rabbit IgG-HRP secondary antibody for $1 \mathrm{~h}$ at room temperature and visualized using an enhanced chemiluminescence detection system.

\section{Proteomics}

SDS-PAGE and detection by MS compatible silver staining. MCF7/WT and MCF7/MX cells were fractionated using the ProteoExtract ${ }^{\circledR}$ Subcellular Proteome Extraction kit (Merck KGaA, Darmstadt, Germany). Protein samples (5 $\mu \mathrm{g}$ ) were separated by SDS-PAGE. Upon completion, the gel was transferred to a clean and dry glass chamber for silver staining using the PlusOne Silver Staining kit, Protein (GE Healthcare, Hino, Japan).

Identification of protein. In-gel digestion using trypsin was performed as previously described (11). The tryptic peptides were used for identification of proteins by mass spectrometry. The digested peptides were analyzed using a nanoflow LC-MS/MS system with a direct nanoflow LC system (DiNa; KYA Technologies, Tokyo, Japan) and a LTQ Orbitrap XL-ETD mass spectrometer (Thermo Fisher Scientific, Inc., Waltham, MA, USA). Data were searched against NCBI human sequence database using Mascot (Matrix Science, Ltd., London, UK).

Statistical analysis. The results are expressed as means \pm SD. Each value represents the mean \pm SD of two or three independent experiments. One-way analysis of variance (ANOVA) with Dunnett multiple comparison test and t-test were performed, and $\mathrm{P}<0.01$ was considered to indicate a statistically significant difference.

\section{Results and Discussion}

$N P-1250$ is an ABCG2 inhibitor and treatment alone reduces the cell viability of ABCG2-overexpressing MCF7/MX cells. We measured ATP-dependent $\left[{ }^{3} \mathrm{H}\right] \mathrm{MTX}$ transport in plasma membrane vesicles, prepared from ABCG2-expressing Sf9 cells, in the presence of a potassium salt of NP-1250. Potassium salt of NP-1250 inhibited ABCG2-mediated MTX transport in a dose-dependent manner (Fig. 1A). This degree of inhibition was comparable to gefitinib (9), suggesting that NP-1250 is a powerful ABCG2 inhibitor. To examine whether NP-1250 overcomes ABCG2-mediated resistance, the cytotoxic effect of ABCG2-substrate SN-38 in the presence of NP-1250 on Flp-In-293/ABCG2 cells was measured. NP-1250 dosedependently reversed SN-38 resistance in Flp-In-293/ABCG2 cells (Fig. 1B). We then evaluated NP-1250 on MCF7/MX cells, a mitoxantrone-resistant cell line in which ABCG2 was amplified and overexpressed (Fig. 2) by chronic exposure to mitoxantrone. Differing from our expectation, NP-1250 exhibited no reversing effect on MCF7/MX cells at concentrations $<20 \mu \mathrm{M}$ (Fig. 1C). Notably, $20 \mu \mathrm{M}$ of NP-1250 inhibited cell viability with a very low dose of $\mathrm{SN}-38$. These results suggest that ABCG2-inhibitor NP-1250 itself may have a cytotoxic effect.

NP-1250 induces apoptotic cell death in MCF7/MX cells. We compared the chemosensitivity to NP-1250 of drug-sensitive MCF7/WT cells and multidrug-resistant MCF7/MX cells by examining the cell viability and cell morphological changes. We discovered that NP-1250 alone reduced the cell viability of MCF7/MX cells, notably, more than that of MCF7/WT cells (Fig. 3A). For the cell morphological study, the number of MCF7/MX cells was significantly less than the number of cells in the control and the adherent cells displayed large vacuoles in the cytoplasm, exhibited atrophic changes or were almost dying (Fig. 3B). In contrast, little change was observed in MCF7/WT cells after a 72-h treatment (Fig. 3B). To confirm these initial observations, we analyzed apoptosis by TUNEL assay and phosphatidylserine (PS) externalization. After a 48-h treatment with NP-1250, in MCF7/MX cells, the number of TUNEL-positive cells was elevated (Fig. 3C) and the TUNELpositive rate was significantly increased in a dose-dependent manner (Fig. 3D). Apoptotic cell death observed in the MCF7/ MX cells upon treatment with NP-1250 was further confirmed by determining Annexin V binding to PS translocated from the inner to the outer membrane surface of the plasma membrane, a hallmark of early stage apoptosis (Fig. 3E).

NP-1250-induced cell death is 'caspase-independent apoptosis'. After $48 \mathrm{~h}$ of treatment with NP-1250, cleavage of PARP was detected using an antibody recognizing the $89-\mathrm{kDa}$ fragment (Fig. 4A). Although PARP is a well-known substrate of 

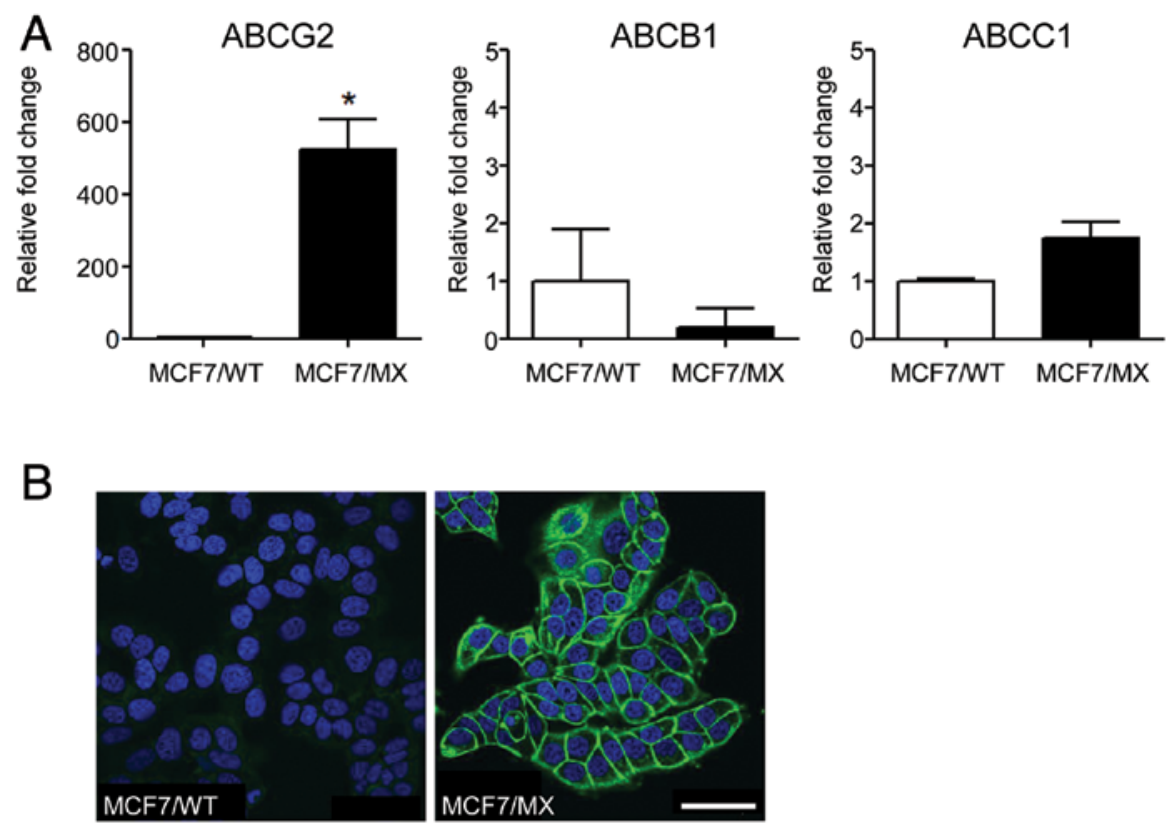

Figure 2. Expression of ABC transporters in human breast carcinoma MCF7/WT cells and multidrug-resistant MCF7/MX cells. (A) ABCG2, ABCB1 and ABCC1 mRNA expression as assessed by real-time PCR. MCF7/MX cells expressed a significantly increased level of ABCG2 mRNA ( $\sim 500$-fold) relative to MCF7/WT cells. Expression was normalized against the housekeeping gene GAPDH. Columns, means; bars, SD ( $\mathrm{n}=3$; $\left.{ }^{*} \mathrm{P}<0.01\right)$. (B) Immunofluorescence of ABCG2. Highly expressed ABCG2 localized almost entirely at the cell membranes in MCF7/MX cells. Scale bar, $50 \mu \mathrm{m}$.

caspases, particularly caspase-3 and caspase-7, MCF7 cells do not express caspase- 3 due to the deletion of 125 nucleotides in the CASP-3 mRNA (12). We confirmed the lack of caspase-3 protein in these MCF7/WT and MCF7/MX cells (data not shown). We, therefore, examined whether caspase-7 and caspase-9 were cleaved prior to cleavage of PARP, but we did not detect either one (Fig. 4A). To further ascertain whether NP-1250-induced apoptosis of MCF7/MX cells was caspase-dependent or -independent, MCF7/MX cells were pretreated with a caspase inhibitor prior to NP-1250 treatment, and cleavage of PARP was subsequently examined. Pretreatment with the pan-specific inhibitor completely abolished the cleavage of PARP, and the pharmacological inhibitor specific for caspase-3/7 also showed a significant inhibition of the cleavage of PARP (Fig. 4B). Under the same experimental conditions, pretreatment of MCF7/MX cells with these inhibitors did not block the production of apoptotic cells (Fig. 4C). These results suggest that caspases, which cleave PARP, are cleaved and activated upon exposure of MCF7/MX cells to NP-1250 but that this is not the main apoptotic cascade in the death of MCF7/MX cells.

To evaluate the effects of NP-1250 on other MDR cell lines that exhibit increased expression of $\mathrm{ABC}$ transporters, we treated several MDR cell lines and their drug-sensitive parental cell lines with NP-1250. We did not observe growth inhibition by NP-1250 in any of the cell lines (data not shown). We also treated several other cancer cell lines, specifically human hepatocellular carcinoma cell line $\mathrm{HuH7}$, human cervical adenocarcinoma cell line HeLa and human mesothelioma cell lines H226 and MESO4, with NP-1250, but we did not confirm any effect (data not shown). Taken together, these results suggest that the antiproliferative/apoptotic effect of NP-1250 may be limited to MCF7/MX cells.
Search for target proteins by proteomics. There are no reports relating inhibition of $\mathrm{ABC}$ transporters to cell death, therefore, we speculated that NP-1250 may have other targets in MCF7/ MX cells.

We excised several bands that were observed in the protein electrophoretograms of MCF7/MX cells. As a control, we also excised the band that appeared to be ABCG2, $74 \mathrm{kDa}$, as observed in the membrane/organelle fraction of MCF7/MX cells. ABCG2 was identified in this band with a Mascot score of 192, thus we excluded proteins whose Mascot scores were $<100$. We also excluded proteins observed at positions $>15 \mathrm{kDa}$ different from their original molecular weight. Candidate proteins that passed our identification criteria are listed in Table II. We selected molecules preferentially involved in cell growth and survival from these proteins and performed realtime PCR to confirm whether the expression of genes encoding these proteins was increased in the MCF7/MX cells when compared to the MCF7/WT cells. Among the 46 proteins, we analyzed the gene expression of 22 proteins, including ABCG2. Three of these proteins, peroxiredoxin-2 (PRDX2), protein disulfide-isomerase A4 (PDIA4) and prohibitin-2 (PHB2), showed significantly elevated mRNA expression in MCF7/MX cells compared to the expression levels in MCF7/ WT cells (Fig. 5). PDIA4 is a protein disulfide isomerase (PDI), an enzyme that catalyzes disulfide formation and isomerization and a chaperone that inhibits aggregation (13) and a member of a large family of dithiol/disulfide oxidoreductases, the thioredoxin superfamily. While it has been reported that PDI shows an inverse correlation with resistance (14), Liu et al reported that increased expression of PDI was found in multidrug-resistant MCF7/AdVp3000 cells when compared to MCF7 cells (15). PHB2, known as a repressor of estrogen receptor (ER) activity, has been shown to interact with and 

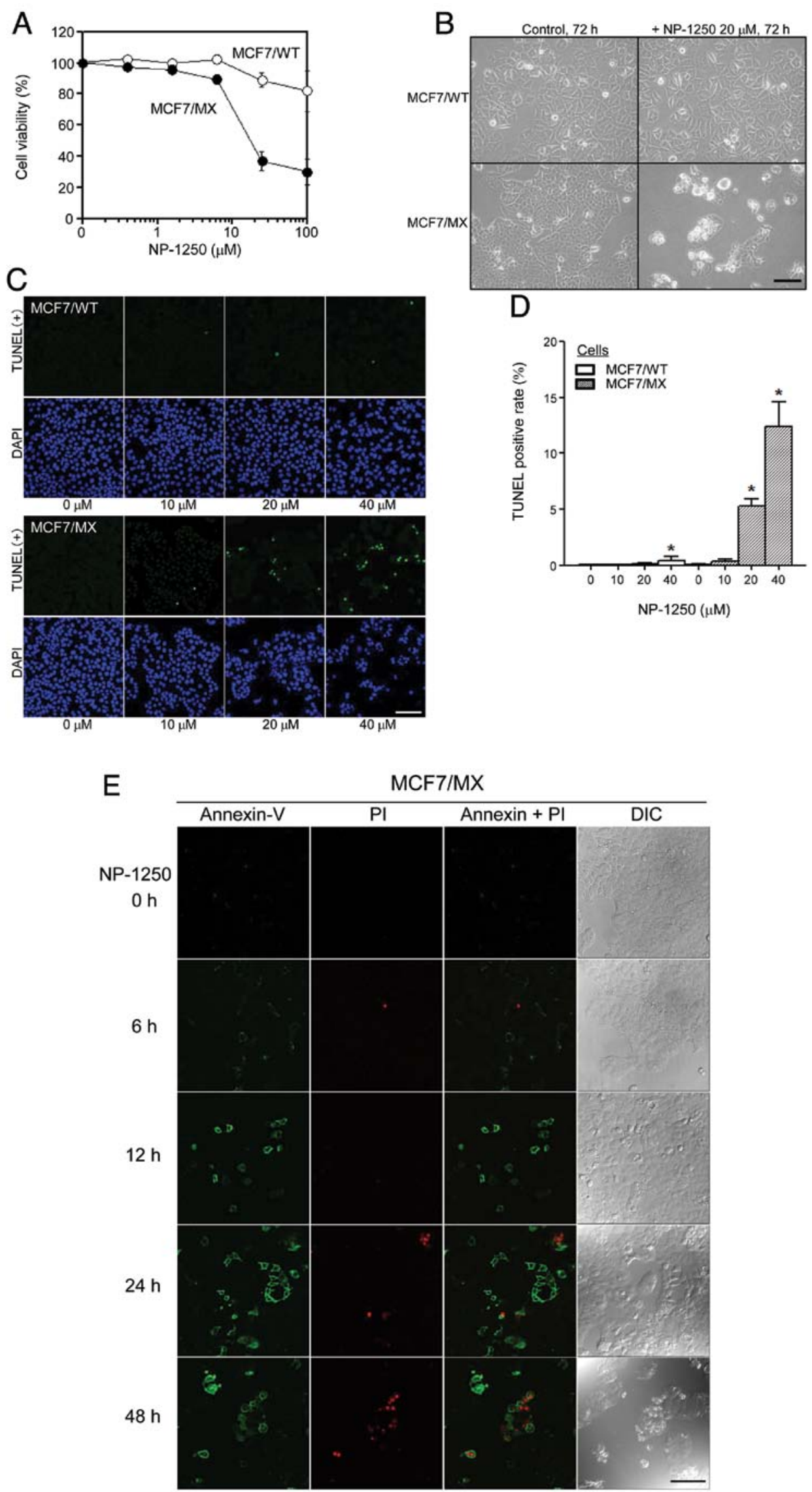

Figure 3. Effect of NP-1250 on cell growth and apoptosis of MCF7/WT and MCF7/MX cells. (A) Dose response effects of NP-1250 on cell viability. Data points, means; bars, $\mathrm{SD}(\mathrm{n}=3)$. (B) Cell morphological changes after a 72-h incubation with $20 \mu \mathrm{M}$ of NP-1250. Scale bar, $200 \mu \mathrm{m}$. (C) TUNEL staining after a 48-h incubation with NP-1250. Scale bar, $100 \mu \mathrm{m}$. (D) TUNEL-positive ratio calculated as described. Columns, means; bars, SD ("P<0.01). (E) MCF7/ MX cells incubated with $20 \mu \mathrm{M}$ of NP-1250 were examined for Annexin V/PI staining. Cells undergoing early stage apoptosis were visualized by binding of (fluoresein-conjugated) Annexin V to translocated phosphatidylserine (green). Cells with a ruptured plasma membrane in the process of cell death were visualized by binding of propidium iodide (PI) to cellular nucleic acid (red). Scale bar, $100 \mu \mathrm{m}$. 


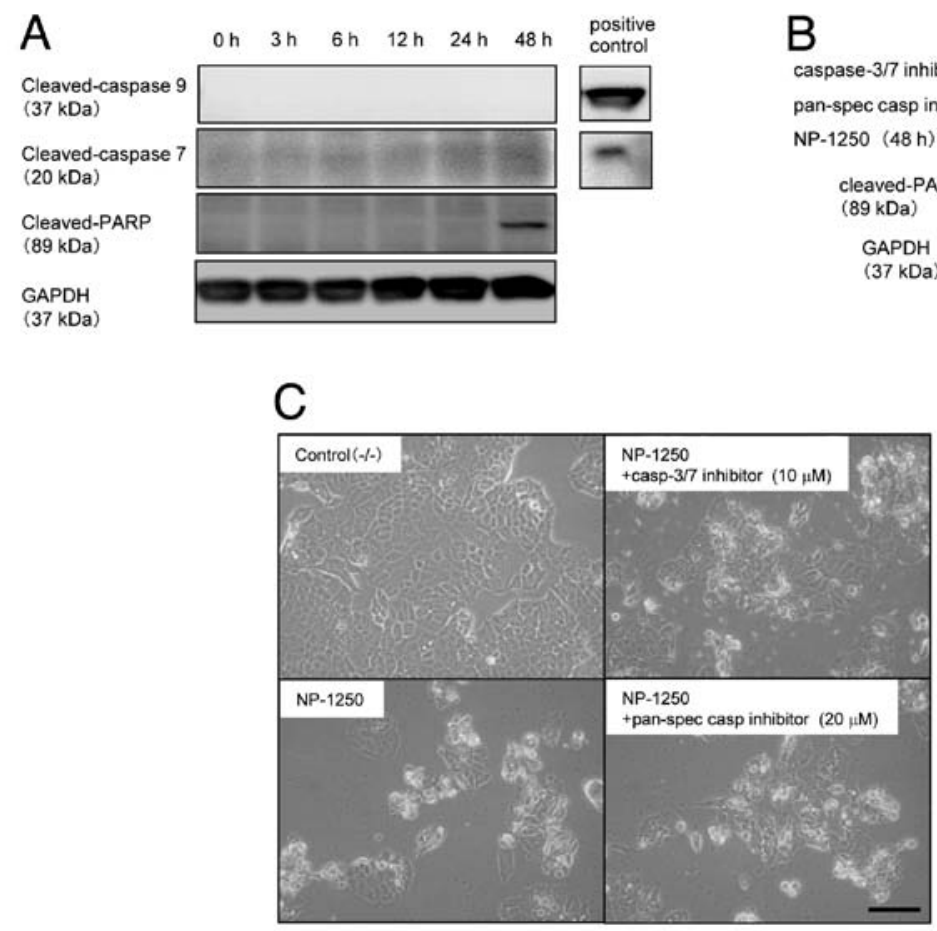

Figure 4. NP-1250-induced apoptosis of MCF7/MX cells involves caspase activation but is 'caspase-independent'. (A) Induction of the cleavage of PARP, a substrate for caspase-3/7, in NP-1250-treated MCF7/MX cells. GAPDH was used as a loading control. Positive controls: cleaved-caspase-9, protein lysate of HeLa cells treated with $1 \mathrm{mM}$ staurosporine for $6 \mathrm{~h}$; cleaved-caspase-7, protein lysates of MCF7 cells treated with $1 \mathrm{mM}$ staurosporine for $3 \mathrm{~h}$. Pretreatment with caspase-3/7 inhibitor or pan-specific inhibitor led to significant reduction in PARP cleavage (B) but did not block NP-1250-induced apoptotic cell death (C). Scale bar, $100 \mu \mathrm{m}$.
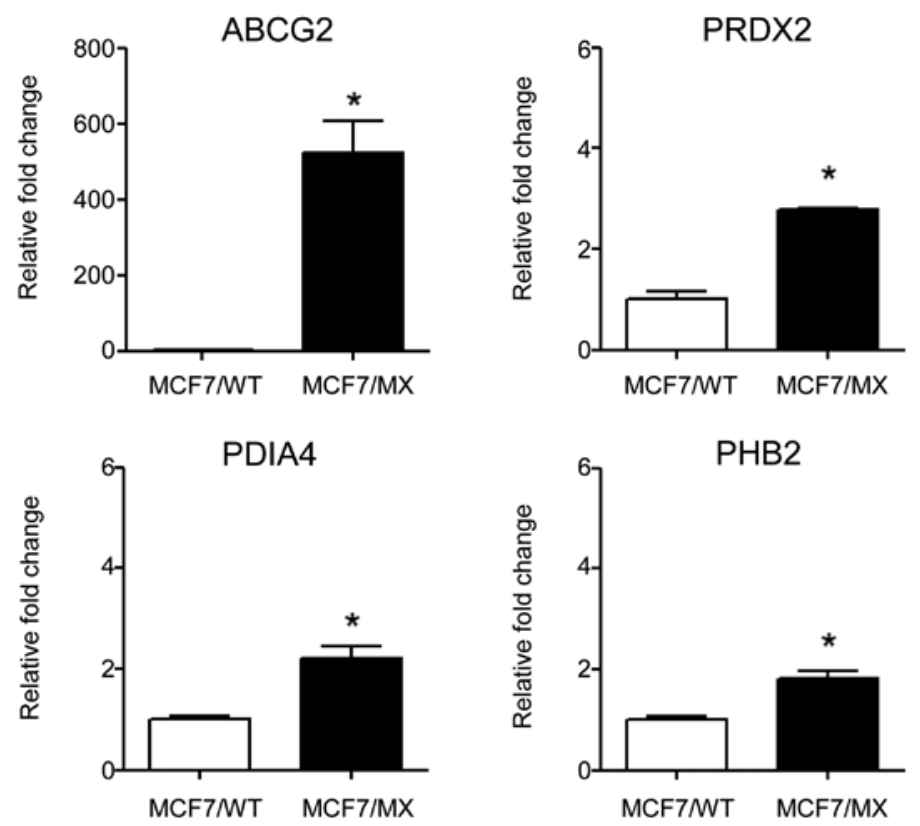

Figure 5. Proteins whose mRNAs were elevated in MCF7/MX cells. ABCG2, ATP-binding cassette, subfamily G, member 2. PRDX2, peroxiredoxin-2. PDIA4, protein disulfide-isomerase A4. PHB2, prohibitin-2. Columns, means; bars, $\mathrm{SD}$ ( $\mathrm{n}=3$; $\mathrm{P}<0.01)$.

inhibit the transcriptional activity of the ER (16). While PHB2 has been implicated in a previously uncharacterized pathway of multidrug resistance in Caenorhabditis elegans (17), Keenan et al found that PHB2 was inversely correlated with resistance in a squamous lung cancer cell line (14). PRDX2 has been previously known as a natural killer-enhancing factor B (18) and is induced by various oxidative stimuli. It plays an important protective role against oxidative radical damage by reactive oxygen and nitrogen species (19). Its expression is correlated with resistance to apoptosis induced by radiation 
Table II. The candidate target proteins of NP-1250 identified by mass spectrometry.

\begin{tabular}{|c|c|c|c|c|c|}
\hline No. & Prot_acc ${ }^{\mathrm{a}}$ & Identified protein $^{\mathrm{a}}$ & Prot_pI ${ }^{b}$ & Prot_mass ${ }^{\mathrm{b}}$ & Prot_score ${ }^{c}$ \\
\hline 1 & PRDX2_HUMAN & Peroxiredoxin-2 & 5.66 & 22,091 & 533 \\
\hline 2 & RL9_HUMAN & 60S ribosomal protein L9 & 9.96 & 21,992 & 274 \\
\hline 3 & RL18_HUMAN & $60 \mathrm{~S}$ ribosomal protein $\mathrm{L} 18$ & 11.73 & 21,763 & 232 \\
\hline 4 & RAB7A_HUMAN & Ras-related protein $\mathrm{Rab}-7 \mathrm{a}$ & 6.40 & 23,830 & 191 \\
\hline 5 & RS5_HUMAN & 40S ribosomal protein S5 & 9.73 & 23,075 & 256 \\
\hline 6 & LGUL_HUMAN & Lactoylglutathione lyase & 5.12 & 21,048 & 145 \\
\hline 7 & RHOA_HUMAN & Transforming protein RhoA & 5.83 & 22,180 & 184 \\
\hline 8 & COMD3_HUMAN & COMM domain-containing protein 3 & 5.63 & 22,421 & 118 \\
\hline 9 & RAB10_HUMAN & Ras-related protein Rab-10 & 8.59 & 22,811 & 150 \\
\hline 10 & PRDX1_HUMAN & Peroxiredoxin-1 & 8.27 & 22,380 & 146 \\
\hline 11 & GLTP_HUMAN & Glycolipid transfer protein & 6.90 & 24,048 & 112 \\
\hline 12 & TAGL2_HUMAN & Transgelin-2 & 8.41 & 22,590 & 648 \\
\hline 13 & PSB5_HUMAN & Proteasome subunit $\beta$ type- 5 & 6.43 & 28,675 & 420 \\
\hline 14 & PEBP1_HUMAN & Phosphatidylethanolamine-binding protein 1 & 7.01 & 21,186 & 366 \\
\hline 15 & RS7_HUMAN & 40S ribosomal protein $\mathrm{S} 7$ & 10.09 & 22,113 & 248 \\
\hline 16 & RS9_HUMAN & 40S ribosomal protein $\mathrm{S} 9$ & 10.66 & 22,649 & 262 \\
\hline 17 & GGCT_HUMAN & $\gamma$-glutamylcyclotransferase & 5.07 & 21,278 & 185 \\
\hline 18 & GNA1_HUMAN & Glucosamine 6-phosphate N-acetyltransferase & 8.17 & 21,162 & 147 \\
\hline 19 & DCTP1_HUMAN & dCTP pyrophosphatase 1 & 4.93 & 18,811 & 105 \\
\hline 20 & RCL_HUMAN & Deoxyribonucleoside 5'-monophosphate N-glycosidase & 4.97 & 19,239 & 110 \\
\hline 21 & DCD_HUMAN & Dermcidin & 6.08 & 11,419 & 100 \\
\hline 22 & LDHA_HUMAN & L-lactate dehydrogenase A chain & 8.44 & 37,021 & 566 \\
\hline 23 & PCNA_HUMAN & Proliferating cell nuclear antigen & 4.57 & 29,177 & 346 \\
\hline 24 & RL5_HUMAN & 60S ribosomal protein L5 & 9.73 & 34,625 & 333 \\
\hline 25 & IPYR_HUMAN & Inorganic pyrophosphatase & 5.54 & 33,207 & 284 \\
\hline 26 & RL6_HUMAN & 60S ribosomal protein L6 & 10.59 & 32,779 & 289 \\
\hline 27 & RA1L3_HUMAN & Putative heterogeneous nuclear ribonucleoprotein A1-like 3 & 9.23 & 34,415 & 180 \\
\hline 28 & OTUB1_HUMAN & Ubiquitin thioesterase OTUB1 & 4.85 & 31,549 & 176 \\
\hline 29 & CHIP_HUMAN & E3 ubiquitin-protein ligase CHIP & 5.61 & 35,403 & 110 \\
\hline 30 & AN32E_HUMAN & $\begin{array}{l}\text { Acidic leucine-rich nuclear phosphoprotein } 32 \text { family } \\
\text { member E }\end{array}$ & 3.77 & 30,958 & 106 \\
\hline 31 & PP4C_HUMAN & Serine/threonine-protein phosphatase 4 catalytic subunit & 4.91 & 35,768 & 115 \\
\hline 32 & NACA_HUMAN & Nascent polypeptide-associated complex subunit $\alpha$ & 4.52 & 23,370 & 117 \\
\hline 33 & PHB2_HUMAN & Prohibitin-2 & 9.83 & 33,276 & 489 \\
\hline 34 & VDAC2_HUMAN & Voltage-dependent anion-selective channel protein 2 & 7.49 & 32,186 & 319 \\
\hline 35 & ROA1_HUMAN & Heterogeneous nuclear ribonucleoprotein A1 & 9.17 & 38,865 & 238 \\
\hline 36 & P5CR1_HUMAN & Pyrroline-5-carboxylate reductase 1 , mitochondrial & 7.18 & 33,624 & 356 \\
\hline 37 & EFTS_HUMAN & Elongation factor Ts, mitochondrial & 8.62 & 35,794 & 157 \\
\hline 38 & ELAV1_HUMAN & ELAV-like protein 1 & 9.23 & 36,282 & 143 \\
\hline 39 & SFXN1_HUMAN & Sideroflexin-1 & 9.22 & 35,952 & 108 \\
\hline 40 & PDIA4_HUMAN & Protein disulfide-isomerase A4 & 4.96 & 73,313 & 1,623 \\
\hline 41 & HNRPM_HUMAN & Heterogeneous nuclear ribonucleoprotein $\mathrm{M}$ & 8.84 & 77,819 & 566 \\
\hline 42 & ECHA_HUMAN & Trifunctional enzyme subunit $\alpha$, mitochondrial & 9.16 & 83,870 & 331 \\
\hline 43 & ABCG2_HUMAN & ATP-binding cassette sub-family G member 2 & 8.91 & 73,120 & 192 \\
\hline 44 & GPDM_HUMAN & Glycerol-3-phosphate dehydrogenase, mitochondrial & 7.57 & 81,441 & 262 \\
\hline 45 & TRAP1_HUMAN & Heat shock protein $75 \mathrm{kDa}$, mitochondrial & 8.30 & 80,415 & 117 \\
\hline 46 & GRP78_HUMAN & Glucose-regulated protein $78 \mathrm{kDa}$ & 5.07 & 72,431 & 248 \\
\hline
\end{tabular}

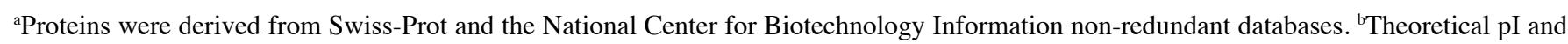
molecular weight obtained from Swiss-Prot. ${ }^{\mathrm{c}}$ MASCOT score for the identified proteins based on the peptide ion score. 
therapy or anticancer drugs (20), highlighting the potential clinical importance of PRDX2 in chemotherapy resistance in cancer. However, the association of PRDX2 and MCF7/MX cells has not been previously reported. Thus further studies, such as knockdown experiments, are warranted.

In the present study, NP-1250 showed no cytostatic effect on other MDR cancer cell lines. However, it may be effective against specific tumors that are clinically similar to MCF7/MX cells and there is still a possibility that it may lead to the identification of new therapeutic molecules for MDR cancers.

\section{Acknowledgements}

We thank Dr Takaichi Hamano (Nippon Chemiphar Co., Ltd.) for the helpful discussions. This study was supported by a Grant-in-Aid for Cancer Research and Grants-in Aid for Scientific Research from the Ministry of Education, Culture, Sports, Science and Technology of Japan, and the Ministry of Health, Labor and Welfare of Japan.

\section{References}

1. Scheffer GL, Maliepaard M, Pijnenborg AC, et al: Breast cancer resistance protein is localized at the plasma membrane in mitoxantrone- and topotecan-resistant cell lines. Cancer Res 60: 2589-2593, 2000.

2. Kuo MT: Roles of multidrug resistance genes in breast cancer chemoresistance. Adv Exp Med Biol 608: 23-30, 2007.

3. Guo X, Ma N, Wang J, Song J, Bu X, Cheng Y, et al: Increased p38-MAPK is responsible for chemotherapy resistance in human gastric cancer cells. BMC Cancer 8: 375, 2008.

4. Honma K, Iwao-Koizumi K, Takeshita F, Yamamoto Y, Yoshida T, Nishio K, et al: RPN2 gene confers docetaxel resistance in breast cancer. Nat Med 14: 939-948, 2008.

5. Katoh SY, Ueno M and Takakura N: Involvement of MDR1 function in proliferation of tumour cells. J Biochem 143: 517-524, 2008.

6. Chen Z, Liu F, Ren Q, Zhao Q, Ren H, Lu S, et al: Suppression of ABCG2 inhibits cancer cell proliferation. Int J Cancer 126: $841-851,2010$

7. Nakagawa M, Schneider E, Dixon KH, Horton J, Kelley K, Morrow $\mathrm{C}$ and Cowan KH: Reduced intracellular drug accumulation in the absence of P-Glycoprotein (mdr1) overexpression in mitoxantrone-resistant human MCF-7 breast cancer cells. Cancer Res 52: 6175-6181, 1992.
8. Wakabayashi K, Nakagawa H, Adachi T, Kii I, Kobatake E, Kudo A and Ishikawa T: Identification of cysteine residues critically involved in homodimer formation and protein expression of human ATP-binding cassette transporter ABCG2: a new approach using the flp recombinase system. J Exp Ther Oncol 5: 205-222, 2006.

9. Saito H, Hirano H, Nakagawa H, Fukami T, Oosumi K, Murakami K, et al: A new strategy of high-speed screening and quantitative structure-activity relationship analysis to evaluate human ATP-binding cassette transporter ABCG2-drug interactions. J Pharmacol Exp Ther 317: 1114-1124, 2006.

10. Wang T, Kajino K, Abe M, Tan K, Maruo M, Sun G, et al: Suppression of cell death by the secretory form of N-terminal ERC/mesothelin. Int J Mol Med 26: 185-191, 2010.

11. Fujimura T, Shinohara Y, Tissot B, Pang PC, Kurogochi M, Saito S, et al: Glycosylation status of haptoglobin in sera of patients with prostate cancer vs. benign prostate disease of normal subjects. Int J Cancer 122: 39-49, 2008.

12. Janicke RU, Sprengart ML, Wati MR and Porter AG: Caspase-3 is required for DNA fragmentation and morphological changes associated with apoptosis. J Biol Chem 273: 9357-9360, 1998.

13. Wilkinson B and Gilbert HF: Protein disulfide isomerase. Biochim Biophys Acta 1699: 35-44, 2004.

14. Keenan J, Murphy L, Henry M, Meleady P and Clynes M: Proteomic analysis of multidrug-resistance mechanisms in adriamycin-resistant variants of DLKP, a squamous lung cancer cell line. Proteomics 9: 1556-1566, 2009.

15. Liu Y, Liu H, Han B and Zhang JT: Identification of 14-3-3sigma as a contributor to drug resistance in human breast cancer cells using functional proteomic analysis. Cancer Res 66: 3248-3255, 2006.

16. Montano MM, Ekena K, Delage-Mourroux R, Chang W, Martini P and Katzenellenbogen BS: An estrogen receptor-selective coregulator that potentiates the effectiveness of antiestrogens and represses the activity of estrogens. Proc Natl Acad Sci USA 96: 6947-6952, 1999.

17. Zubovych I, Doundoulakis T, Harran PG and Roth MG: A missense mutation in Caenorhabditis elegans prohibitin 2 confers an atypical multidrug resistance. Proc Natl Acad Sci USA 103: 15523-15528, 2006.

18. Shau $\mathrm{H}$ and Kim A: Identification of natural killer enhancing factor as a major antioxidant in human red blood cells. Biochem Biophys Res Commun 199: 83-88, 1994.

19. Kowaltowski AJ, Netto LE and Vercesi AE: The thiol-specific antioxidant enzyme prevents mitochondrial permeability transition evidence for the participation of reactive oxygen species in this mechanism. J Biol Chem 273: 12766-12769, 1998.

20. Chung YM, Yoo YD, Park JK, Kim YT and Kim HJ: Increased expression of peroxiredoxin II confers resistance to cisplatin. Anticancer Res 21: 1129-1133, 2001. 